- paciente não fazia lavagens com antisepticos, mes se limitava a applicar um tratamento que nos pareceu o mais adequado, qual o do emprego da pomada, sem que houvesse o menor cuidado, de asseio siquér, da região.

$3 .^{\circ}$ ) Não nos parece que os ferimentos da mão esquerda tenham produzido privação permanente do uso do membro superior esquerdo. A flexão da phalangeta sobre a phalanginha e desta sobre a phalange não se faz no medio e é muito incompleta no indicador e no anular.

Mas quér nos parecer que a condição actual se modificará basrante com o tempo. Estamos convencidos de que com o tratamento adequado a muito pouco se reduzirá a diminuicãa da capacidade funccional dos dedos que actualmente existe.

Não nos parece que tivesse havido secção dos ten'dões flexores no indicador e no annullar e si secção houve no medio, não foi completa. A incapacidade actual na flexão dos dedos depende, a nosso ver, de não estarem ainida de todo consolidedos os ferimentos. Assim, na peor das hypotheses, resultará da lesão, para o offendido, diminuição nos movimentos de flexão das phalangetas sobre as phalanginhas e destas sobre as phalanges, mais accentuada no medio do que nos outros dedos. Ainda assim, haverá diminuição na capacidade funccional na mão esquerda do offendido, e não privação permanente do uso della, mesmo tendo em vista a profissão a que se entregava antes de cegar.

\title{
V
}

De accôrdo com este nosso juizo, respondemos aos quesitos:

Ao $1 .^{\circ}$ : Não.

Ao $2 .^{\circ}$ : Sim.

Ao $3 .{ }^{\circ}$ : Bom.

DR. OSCAR FREIRE.

DR. FLAMINIO FAVERo.

\section{PERDAS DE PHOSPHATOS}

SUMMARIO :. Deposito branco expontaneo. Emisão de urinu branca leitosa. Augmento absoluto da dosagem dos phosphatos. Precipitação pelo calor. Significação do phosphato ammoniaco-magnesiano. Relação urologica do phosphoro $e$ azoto. Metabolismo do phosphoro. Importancia do phosphoro. Os 4 grupos: phosphatos iñnorganicos, proteinas phosphoradas. gorduras phosphoradas $e$ hydratos de carbono phosphorados. Relação existente entre as differentes formas phosphoradas. Quantidade de phosphoro necessaria ao organismo. Excrę̧ão do phospnoro. Urigemi aos phosphatos urinarios.

Não é infrequente recebelr-se no consultorio um doente que procura o medico por soffrer, diz elle, de perdas de phosphatios, e que 
apezar da medicação constituida por preparados phosphorados não melhorra de sua phosphaturia. São em geral individuos de uma certa culitura, apresentando sympitomas neurasthenicos; esses individuos trazem, habitualmente, tres ou mais analyses de urina nas quaes se verifica a existencia de trez, quatro ou mais grammas de phosphatos.

Como chegou esse dioente ao diagnosticos de phosphaturia? Fol ou por si ou pello medico. O proprio doente teve a sua attenção despertada ou porque era espessa e esbranquicenta semelhando ao leite quando emittilda; manidou elle mesmo examinar a urina e a analyse revelou que o deposito era constituido de phosphertos.

Outras vezes o diagnostico é feito pelo medico que verificiando uma taxa mais elevada que a normal, diagnostica uma phosphaturia. Esse diagnositico em geral satisfaz o doente, pois é do dominio geral a imporitancia do phosphoro na constituição do systema nervoiso e ldos elementos sexuaes; essa perda de phosphaito lhe dá uma explicação para a sua miemıria fraca, cansaço facil, perdas seminaes, etc.; e miesmo o proprio medico sabendo da compiosição das nucleoproteinas e das phosphatides e de sua importancia no metabolismo é elle mesmo levaido a comsidenar o phosphoro eliminado como a expressâo do metabolinismo das nucleoproteinas e das phosphatides, como o azoto é tomado como medida do metablismo das proteinas em geral; veriemos adeanitie o valor dessias hypotheses siobre o metabolismo do phosphoro. Comı o doente é um restudante, um advogrado, um professor, portanito individuos que trabalham com o cerebro ou é um individuo que se tem entregado a excessos venereos ou tem tido poluções nocturnas ou se trata de um neurasthenico, essa hypothese torna-se ainđda mais symparthica e parece então logica a adminilstração de preparados phossphatados para comibater esisa pseudo perda de phosphaitos. A consequencia dessia administração é naturalmente augmentar a eliminaçĩo dos phosphatos.

Vamıs encarar as differentes modialidiades pelas quaes o doemte ou o medico chegam ao diagnostico de perda de phosphatos e terminaremos dizendo algumas pallavras sobre o metabolismo do phosphoro.

1.o - A urina resfriando deixa um deposito branco de phosphatos. - Esita precipitação dos phosphatos em geral não indica um augmento de phosphatos; indica apenas que a urina é neutra ou alcalina, o que faz precipitar os phosphatos menos soluveis de calcio e meagnesio ou phosphatos terrosos. Ora, esse deposito de phosphato é um processo puramente physiologico. Uma mollecula de acido phostphorico ( $\left.\mathrm{H}^{3} \mathrm{PO}^{4}\right)$, contendo trez atomos de hydrogenio, cada um delles póide ser substituido por um atomo de qualquer metal monoatomico, tal como o sodio, por exemplo. Podem-se formar trez typos de saes conforme um, dois ou trez atomos sejam substituidos:

$\mathrm{Na} \mathrm{H}^{2} \mathrm{PO}_{4}^{4}$ - Phosphato di-hydrico de sodio ou ortho-phosphato monometalico ou phosphato acido de sodio.

$\mathrm{Na} 2 \mathrm{HOP} 4$ - Phosphato monohyldrico ou ortho-phosphato dimetalico ou phophato neutro de sodio.

Na3PO4 - Phosphato de sodio ou phosphato tri-metalico ou phosphato basico de sodio.

A differença enitre estes trez phosphatios se refere á sua acção sobre o papel Turnesol e á sua solubilidade. 0 phosphatio acido de sodio tocina vermelho o papel azul; a acidez da urina é principarlmente devida á elle. E' o mails soluvel dos trez. $O$ phosphato mono- 
hydrico, $\mathrm{Na}^{2} \mathrm{HPO}^{4}$, é tambem technicamente falando um sal acido, mas não reage a o papel turnesol, tanto que quando uma urina contem mais $\mathrm{Na}_{2}^{2} \mathrm{HPO}_{4}^{4}$ do que $\mathrm{NaH}_{2}^{2} \mathrm{PO}^{4}$ essa urina $e ́$ alcalina. Quando a quantidade se equivale a reacção é amphoterica. 0 phosphatio momohydrico é muito menos soluvel que o phosphato acido de sodio. O phosphato trimetalico $\mathrm{Na}^{3} \mathrm{PO}^{4}$ é ainda menos soluvel e tem reacção alcalina ao turnesol. De forma que quando dizemos ser uma urina alcalina quer isto dizer que existe pouco phosphato Goluvel $\mathrm{NaH}^{2} \mathrm{PO}_{4}^{4}$ e muito dos menos soluveis $\mathrm{Na}^{2} \mathrm{HOP} 4$ e Na3PO4. De forma que uma urina alcalina precipita os seus phosphatos não porque os phosphatos em geral sejam mais soluveis nas soluções acidas que nas alcalinas, pois não se trata de solubilidade do mesmo sal; mas a urina acida não precipita os seus posphphatos porque elles estão sob uma forma mais soluvel ( $\left.\mathrm{NaH}^{2} \mathrm{PO}^{4}\right)$, emquanto a alcalina tem os seus phosphatos sob uma forma menos soluvel $\left(\mathrm{Na}^{2} \mathrm{HPO}^{4}\right)$ e $\left(\mathrm{Na}^{3} \mathrm{PO}^{4}\right)$. O que dissemos do phosphato de sodio refere-se a todos cs outros (iphosphatos de magnesio e de calcio). Fstes ultimos sendo menos soluveis são os que maís facilmente precipitam. Agora, perguntarão os senhores: porque uma dada urina contem mais: phosphators insoluveis do que soluveis? Isto dependie muito simplesmente, de um lado, da quantidade die acido phosphicr.ico presente e do outro da quantidade de bases (sodio e potassio etc.), e da quantidade de outros acidos presenter sob a forma de chloretos e sulphatos. Quanto maior for a quantidade de chloretos e sulphatos itanto menor a quantidade de bases dəixad $s$ livres para se combinar com ac.do phosphorico; como consequencia maior porcentagem de $\mathrm{NaH}^{2} \mathrm{PO}^{4}$. Inversamente, quanto menor a quantidade de chloretos e sulphatos maior a quantidade de bases livres e portanto maior a proborção de $\mathrm{Na}^{2} \mathrm{HPO}^{4}$, portanto urina aicalina devido a maior proporção de posphatos menos soluveis ilavendo pois maior probabilidade de precipitação exponitanea de phossphatos. Já vêm os senhores como a precipitação de phosiphartos não indica perdia de phosphatos.

$2 .^{\circ}$ - A urina de muitas pessôas sans e mesmo de creanças é, ás vezes, côr de leite logo apóz uma lauta refeição. Issto é causa de grande alarme por parte do doente. No entanito esta urina é perfeitamente physiojogica e devida a que grande quantidade de $\mathrm{HCl}$ é secretada no estomago para a digestão, dando em resultado que a urina contenha temporariamente grande numero de basies e ois phosnhatos menos soluveis como os de calcio e magnesio precipitam. mesmo no interior da bexiga. E' portanto dependente ainda da alcalinidade da urina.

3.o - A analyse da urina dá uma maior cifra que a media normal. A variação em individuos normaes é muito ampla, de uma a oito grammas, cifras essas maiores que as medias dadas pelas papeletas de laboratorio. 0 augmento de p-osphatos para poder ser aprec.ado deverá ser comparado com a quantidade ingerida com os alimøntos e com a quantidade excretada com as fezes o que exige perqulzas que não estão no dominio da clinica diaria. Um augmento de rhosphatos até ulteriores pesquizas deve ser considerado simplesmente como a expressão de uma allimentação rica em phosphoro, como a carne, óvos, queijo, aveia, feijão, amendoas, etc. Existe apenas uma molestia - dianjos rhophatica - na qual a eliminação de phosphatos es augmentada de um modo persistente $\theta$ independente, até certo ponto, da alinaentação. Nessa phosphaturia permanente a quantidade de phosphatos attinge até 20 grammas 
por dia. Essta nara condição miorbida é accmipanharda de sêde, commagrecimento, polyuria, dores nas costas e nas cadeiras e de ausencia de glycose.

4.0 - Outras vezes não se faz a dosagem dos phosphatos e o diagnostico é feito rapidamente quando o clinico obtem um deposito sob a forma de uma nuvem branca apoz o aquecimento da urina e esse deposito é soluvel no acido acetico diluido. Diz-se então que os phosphatos sendo menos soluveis na urina quente, precipitam-se. Isto não é exacto; o que se dá é o seguinte: A acção do calor não precipita o mesmo phosphato que a urina fria continha, mas provoca a formação de um outro menos soluvel. O phosphato monohydrico de calcio dissocia-se em phosphato di-hydrico e phos. qhato de calcio normal.

$$
\begin{aligned}
& 4 \mathrm{CaHPO}_{4}=\mathrm{Ca}_{3}\left(\mathrm{PO}_{4}\right)_{2}+\mathrm{Ca}\left(\mathrm{H}_{2} \mathrm{PO}_{4}\right)_{2} \\
& \text { solubilidade relativamente } \\
& \text { media insoluvel e precipi- } \\
& \text { tando como nuvem } \\
& \text { relativamente } 0 \\
& \text { mass soluvel } \\
& \text { dos tres }
\end{aligned}
$$

Portanto, esita preclipitação não indica perda de phosphatos.

\section{SIGNIFICAÇÃo DO PHOSPHATO AMMONIACO-MAGNESIANO}

E' insioluvel e precipita-se sob a forma bem conhecida de caixão de diefunto. E' evidente que elle só se forma quando a urina contivér amoniaco. Nós encontramos este phosphato amoniaco-magnesienıo em trez circumstancias: 1.a) - na decomposição ammoniacal da urina, quanido esta fica exposta ao ar, e neste caso o seu valor é apenas indicar esta decomposição; 2. ) - quandio n'esta decomposiçî̃o exterior póde ser excluida a presença do phosphato ammoniaco magnesiano, indica uma inflammação purulenta nas vias urinarias principalmente na bexiga. Neste caso é preciso que encontremos tambem globulos de puz; $3 . a)$ - é preciso porem não esquecer que $n$ urina normal contem ammonia em quantidade sufficiente para que possamos encontrar alguns crystaes de phosphato ammoniaco magnesiano em urinas normaes, independente da purulencia e da decomprosição exterior.

De accordo com o que acabamos de dizer, não tem em geral importancia clinica a diosiagem dos phosphatos; o medico precisa apenas de uma prova de facil execução que permitta reconhecer ise um precipitado é ou não consitituido de phosphato. Isto conseguimos pela stimplles addicção de acido acetico diluido que dissiolve o precipitado formado pelo phosphato, permittindo aio clinuico não confundir: 1.0) com albumina em caso de precipitação pelo calôr, 2.o) com puz em caso de um depositio expontaneo; 3.0 ) com chyluria, corrimento ganorrheico ou espermathorreia, quando a urina é emitida de côn leitossa.

\section{METABolismo do PHOSPHORO}

O phosphoro é largamente distribuido no organismo humano, desempenhinando papel tão importante quanto a proteina. A relação que os compositos do phosphoro têm com as funcções de nutrição são esboçadias nas seguintes linhas por Forbes e Keith: "Nenhum outro elemento inorganico entra em tamanha diversidade de compostios, nem representa um papel tão importante em tanitas funç̧⿸丆⿰丨丶㇀ 
como o phosphoro: Sob o ponto de vista estructural elle faz parte de todos os nucleos cellulares, entrando em parte importante no esqueleto, no leite, nos elementos sexuaes, no tecido glandular, $\theta$ no syistema nervoso. Sob o ponto de vista funccional toma parte em toda a multiplicação cellular, na activação e no "contrôle" das acções dos enzimias, na manutenção da neutralidade do organisımю, na coridiucção dos esitimulos nervosos, e, pelas suas reiraçõłes com a pressão esmotica, a tensão superficial, e a embibição de agua pelos colloides, elle influe sobre os movimentos dos liquidos, sobre o conteu'dio liquido dos tecidos, sobre os movimentos cellularies e sibre a a absolpção te a secreção".

O phosphoro nos alimentos se apresenta em parte sob a forma de phosphatos inorganicos e em parte em combinação com cada um dos trez grupos de substancias organicas, proteinas, gorduras $\theta$ hydratios de carbono. Poidemos pois dividir os compostos phosphorados em quatro grupos:

1.o) Phosphatios inorganicos, dios quaes o die poitasisio é provavelmente o mais abundante nos alimentos, nos fluidos e tecidos molles do organismo, emquanto que o phosphato de calcio é o prinChipad elemento inorganico dos osisos.

$\left.2 .^{\circ}\right)$ Proteinas canitendo phosphoro. São as nucleo-proteinas dos nucleos celulares, as lecitino-proteinas e zals $^{\text {as }}$ veridadeiras phosphoroproteinas como a caseina, o caseinogenio do leite e a ovo-vitelina de gemma de ovo.

$3.0)$ Gorduras phosphoradas são as chamadas phosphatides ou phosipho-lipomas, incluindo nessa classe as lecitinas, lecitan cephalinas, etc. que são enconitradas em grande quantidade no systenia nervoso e em menor quanitidade em toidas as celliules e tecidos do orgurismo. A gemma do ovo contem granıde proporção de phosphatides; poderemos dizer que dois terços do phośphoro da gemma do ovo está sıob a forma de phosphatides.

4.o) Hydratios de carbiono phosphorados. O amido contem phosphoro. Oø saes de potasisio, calcio e magnesio combinidos com o acido phytico diando compostos corihecidos sob o nome de phitatos ou phitinas existentes em todas as sementes, aibundando principalmente no grão do trigo.

\section{REILACT̃O ENTRE AS DIFFERENTES FORMAS PHOSPHORADAS}

Griande numero de pesquizas têm sido feitas para se saber șe os differentes compiostios de phosphoro são utilizados pielo organismo indifferentemente ou se apienas os compostos organicors seriem aproveitadios. Não cansarei a vossa attenção com a enumeração de todas as experiancias feitas, e direi apenas que a conclusão actual é que o organismo animal é capaz de utilizar os phosphatior inorganicos transformando-ós em nucleo proteinas, phosphoproteinas e phosphatides (Hart, Mac Collun, Fuller e Halpin), parecendo apenas que a administração de phosphoro organico é mais favoravel no sentido de ser mais promptamente utilizado; Massilow anha, apóz experiencias proprias, que principalmente durante o periodo de crescimento obtem-se melhores resultados administrando-se o phosphoro soob a forma organica, motivo porque nas dietas das creanças deve-se introđuzir grande porção de leite pela elevada proporção de phosphoro-proteinas e de phosphatides, se bem que em menor quantidade, contidas nesse alimento. 


\section{QUANTIDADE DE PHOSPHORO NECESSARIA AO ORGANISM}

O phosphoro sendo indispensavel á todus os tecilos, o crescimento e renovação delles exige juntamente com a proteina uma certa quantidade de phosphoro. Para se saber qual seja esita quantidade temos que levar em conta a quantidade de phosphoro ingeridra com or alimentos e a quantidade excretada pelas fezes e pelas uninas, pois a relação entre a quantidade excretada pelos intestinois e pelos rins varia dentro de grandes limites. Experiencias assim conduzidas, em 27 individuos, deu em media a quantidade de 0,96 grs. de phosphoro $\left(2,20\right.$ de $\left.\mathrm{P}^{2} \mathrm{O}^{5}\right)$ por 70 kilos de pezo. Dou aqui a quantidade aproximaida de phosphoro em divensos alimentos:

Subsitancias

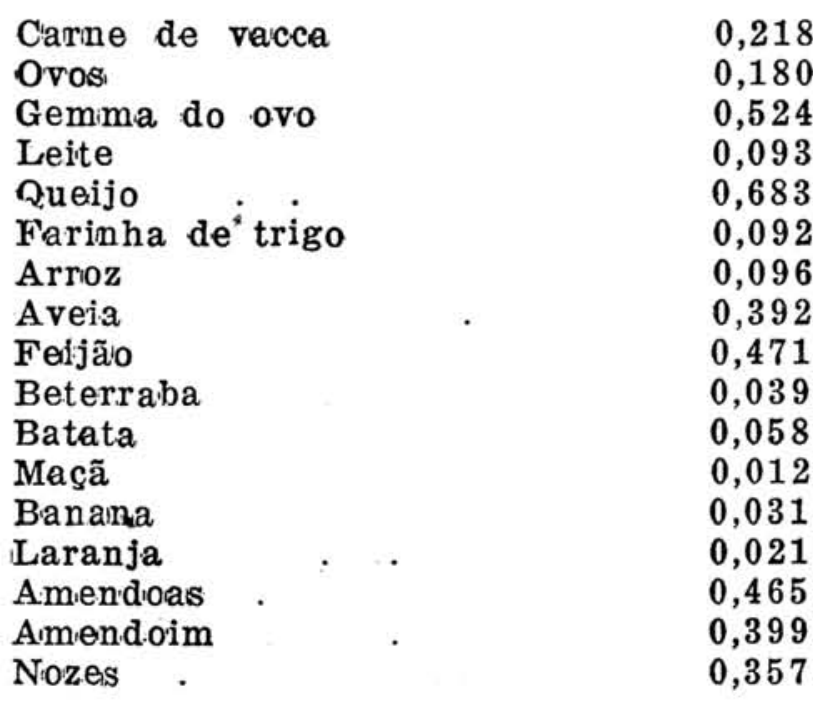

Phosphoro por 100 grs. de substiancias

\section{FXCRECGAO DO PHOSPHORO}

o phosphoro metabolizado é expelido quasi inteiramente sob a forma de phosphatos inorganicos. 0 phosphoro organico da urina é constituido apenas por um a trez por cento do total, sendo que alguns autores duvidam da presença de phosphoro organico na urina. Os carnivoros excretam os phosphatos principalmente pela urina. No homem a quantidade de phosphoro excretada pelos intestínos não póde ser desprezada, pois é bastante consideravel, como tambem não póde ser calioulada a não ser pela meidida exacta das suas quantidades nas fezes; razão pela qual não se póde ter uma ideia exacta do metabolismo do phosphoro apenas pela sua dosagem na urina. O phosphoro é excretado na urina sob a forma de phosphatos alcalinos e terrosos. Os phosphatos alcalinos são constituridos pelos phosphatos de sodto e potassio e constituem dois terços do total. Os phosphatos terrosos constituidos pelos phosphatios de calcio e magnesio, constituem apenas um terço do total sendo excretado em maior quantidade nas fézes. A quantidade de phosphoro excretada por pessóas normaes varia dentro de largo limite; a media é dada em gerall de 3 a 5 gramma por dia, mas de facto os límites normaes de excreção vão de 1 a 8 grammas. 


\section{ORIGEM DOS PHOSPHATOS URINARIOS}

Como o phosphoro dos tecidos existe principalmente sob a forma de nucleophoteinas, e esta é a substancia caracteristica dos nucleocellulares, e porque estes elementios têm um papel importante no mietabolismo, levantou-se a hypothese de que o phosphoro eliminado devia ser o producto do metabolismo das nucleoproteinas. Na realidade, mesmo durante o jejum, o individuo continua a eliminar phosphato, e nesises casos este só poderá ser proveniente do catabolismo cellular; mas a porcentagem de phosphatos attribuida a esia causa é muito diminuta quando comparada com a deriveda dos alimentos ingeridos. Tanto isso é verdade que, no jejum, os phosphatos desapparecem da urina.

Esta pequena porcentagem-de phosphoro proveniente do catabolismo dios tecidos não justificica a hypothese, a saber, que a variação da quantidade de phosphatos na urina seja devido a um augmentado ou diminuido metabolismo do cerebro e tecidos nervosos, representados pelas suas nucleosıproteinas e phophatides, como é crenca entre o vulgo. O facto citado por alguns autores de que as pessoas que têm trabalho intellectual intensio, ou apresienitam periurbaccões neurasthericas, ou excessos sexuaes, excretam m'aior quantidade de phosphatos do que uma pessoa normal, não foi ainda demonstrado sufficientemente. Pois já vimos que a medida dos phosphatos urinarios unicamiente não é basitante para esta concluıão. Além de que as pesquizas de Voit demonstram que o esqueleto do linmem contém 600 grammas de phosphoro, os musiculos 56 grammas e o cerebro e os nervos apenas 5 grammas. Ora, uma predominancia tal em favor do tecido osseo não póde deixar de exercer uma grande influencia sobrre a excreção dos phosphatos derivadios do merabolismo, mesmo admittindo-sio que o metabolismo do trcido bese seja muito menos activo do que o metabolismo do cerebro $\theta$ ãos nervos.

De forma que a principal fonte dos phosphatos urinarios é a alimientação. Uma outra causa é devida aos phosphatos soluvieis no sangue, que represientam um papel muito importante na manutençăı da neutralidade do organismo. Os scnhores sabem que a todo o momento se estão produzindo acidos no organismo, e a neutralização delles se faz pela transformação dos di-phosphatos em mıono-phosphatos que são excretados pela urina.

Resuminido, temos que as fonites dos phosphatos urinarios são: 1.0) - alimentos ingeridos; 2.0) - formação de phosphatos acid.os para manutenção de neutralidade; 3.0) - metabolismo do tecir o osseo (em muito pequena quantidade); 4.0) - metabolismo das nucleoproteinas (em quantidade insignificante).

DR. LEMOS TORRES.

Attesto que tenho empregado em minha clinica o VIDAN com excellentes resultados.

DR. RUBiÃO MEIRA 